\title{
Approximating the erfinv function
}

\author{
Mike Giles*
}

The inverse error function erfinv is a standard component of mathematical libraries, and particularly useful in statistical applications for converting uniform random numbers into Normal random numbers. This chapter presents a new approximation of the erfinv function which is significantly more efficient for GPU execution due to the greatly reduced warp divergence.

\section{Introduction}

Like $\cos x, \sin x, e^{x}$ and $\log x$, the error function

$$
\operatorname{erf}(x)=\frac{2}{\sqrt{\pi}} \int_{0}^{x} e^{-t^{2}} \mathrm{~d} t
$$

and its inverse erfinv $(x)$ are a standard part of libraries such as Intel's MKL, AMD's ACML and NVIDIA's CUDA math library.

The inverse error function is a particularly useful function for Monte Carlo applications in computational finance, as the error function is closely related to the Normal cumulative distribution function

$$
\Phi(x)=\frac{1}{\sqrt{2 \pi}} \int_{-\infty}^{x} e^{-t^{2} / 2} \mathrm{~d} t=\frac{1}{2}+\frac{1}{2} \operatorname{erf}\left(\frac{x}{\sqrt{2}}\right)
$$

so

$$
\Phi^{-1}(x)=\sqrt{2} \operatorname{erfinv}(2 x-1) .
$$

If $x$ is a random number uniformly distributed in the range $(0,1)$, then $y=\Phi^{-1}(x)$ is a Normal random variable, with zero mean and unit variance. Other techniques such as the polar method and Marsaglia's Ziggurat method are usually used to transform pseudo-random uniforms to psuedo-random Normals, but $\Phi^{-1}(x)$ is the preferred approach for quasi-random uniforms

* Oxford-Man Institute of Quantitative Finance, Eagle House, Walton Well Road, Oxford OX2 6ED 
generated from Sobol sequences and lattice methods, as it preserves the beneficial properties of these low-discrepancy sequences [Gla04].

Like trigonometric functions, $\operatorname{erfinv}(x)$ is usually implemented using polynomial or rational approximations [BEJ76, Str68, Wic88]. However, these approximations have been designed to have a low computational cost on traditional CPUs. The single precision algorithm from [BEJ76] which was used in the CUDA 3.0 math library has the form shown in Table 1.

Table 1: Pseudo-code to compute $y=\operatorname{erfinv}(x)$, with $p_{n}(t)$ representing a polynomial function of $t$.

$$
\begin{aligned}
& a=|x| \\
& \text { if } a>0.9375 \text { then } \\
& t=\sqrt{\log (a)} \\
& y=p_{1}(t) / p_{2}(t) \\
& \text { else if } a>0.75 \text { then } \\
& y=p_{3}(a) / p_{4}(a) \\
& \text { else } \\
& y=p_{5}(a) / p_{6}(a) \\
& \text { end if } \\
& \text { if } x<0 \text { then } \\
& y=-y
\end{aligned}
$$

If the input $x$ is uniformly distributed on $(-1,1)$, there is a 0.75 probability of executing the third branch in the code, and only a 0.0625 probability of executing the expensive first branch which requires the computation of $\log (a)$ and its square root.

However, on an NVIDIA GPU with 32 threads in a warp, the probability that all of them take the third branch is $0.75^{32} \approx 0.0001$, while the probability that none of them take the first branch is $0.9375^{32} \approx 0.13$. Hence, in most warps there will be at least one thread taking each of the three branches, and so the execution cost will approximately equal the sum of the execution costs of all three branches.

The primary goal of this paper is to improve the execution speed of 
erfinv $(x)$ through constructing single and double precision approximations with greatly reduced warp divergence, i.e. with most warps executing only one main branch in the conditional code. The technique which is used can be easily adapted to other special functions. The MATLAB code which generates the approximations is provided on the accompanying website, along with the CUDA code for the erfinv approximations, and test code which demonstrates its speed and accuracy.

The efficiency of the new approximations is demonstrated in comparison with the implementations in CUDA 3.0. While this chapter was being written, CUDA 3.1 was released. Its single precision erfinv implementation incorporates the ideas in this chapter, though the code is slightly different to that shown in Table 5. The double precision implementation is still the same as in CUDA 3.0, but a new version is under development.

These approximations were originally developed as part of a commercial maths library providing Numerical Routines for GPUs [NAG09, BTGTW10]. Similar approximations have also been developed independently for the same reasons by Shaw and Brickman [Sha09].

The CUDA source code, the MATLAB code used to create the approximations, and the test code for the evaluation are all freely available [Gil10].

\section{New erfinv approximations}

\subsection{Single precision}

The new single precision approximation erfinv $v_{\mathrm{SP}}$ is defined as

$$
\operatorname{erfinv}_{\mathrm{SP}}(x)=\left\{\begin{array}{ccc}
x p_{1}(w), & w \leq w_{1} & \text { central region } \\
x p_{2}(s), & w_{1}<w & \text { tail region }
\end{array}\right.
$$

where $w=-\log \left(1-x^{2}\right), s=\sqrt{w}$, and $p_{1}(w)$ and $p_{2}(s)$ are two polynomial functions. The motivation for this form of approximation, which in the tail region is similar to one proposed by Strecok [Str68], is that

- erfinv is an odd function of $x$, and has a Taylor series expansion in odd powers of $x$ near $x=0$, which corresponds to $p_{1}(w)$ having a standard Taylor series at $w=0$;

- erfinv is approximately equal to $\pm \sqrt{w}$ near $x= \pm 1$ 
Using $x=\sqrt{1-e^{-w}}$, the left part of Figure 1 plots $\operatorname{erfinv}(x) / x$ versus $w$ for $0<w<16$ which corresponds to the entire range of single precision floating point numbers $x$ with magnitude less than 1 . The right part of Figure 1 plots erfinv $(x) / x$ versus $s \equiv \sqrt{w}$ for $4<w<36$. The extension up to $w \approx 36$ is required later for double precision inputs.

Using polynomials $P_{n}$ of degree $n$, a standard $L_{\infty}$ approximation for the central region would be defined by

$$
p_{1}=\arg \min _{p \in P_{n}} \max _{w \in\left(0, w_{1}\right)}\left|p(w)-\frac{\operatorname{erfinv}(x)}{x}\right| .
$$

However, what we really want to minimise is the relative error defined as $\left(\operatorname{erfinv}_{\mathrm{SP}}(x)-\operatorname{erfinv}(x)\right) / \operatorname{erfinv}(x)$, so it would be better to define $p_{1}$ as

$$
p_{1}=\arg \min _{p \in P_{n}} \max _{w \in\left(0, w_{1}\right)}\left|\frac{x}{\operatorname{erfinv}(x)}\left(p(w)-\frac{\operatorname{erfinv}(x)}{x}\right)\right| .
$$

Since this weighted $L_{\infty}$ minimisation is not possible using MATLAB, $p_{1}$ is instead approximated by performing a weighted least-squares minimisation, minimising

$$
\int_{0}^{w_{1}} \frac{1}{\sqrt{w\left(w_{1}-w\right)}}\left(\frac{x}{\operatorname{erfinv}(x)}\left(p(w)-\frac{\operatorname{erfinv}(x)}{x}\right)\right)^{2} \mathrm{~d} w .
$$
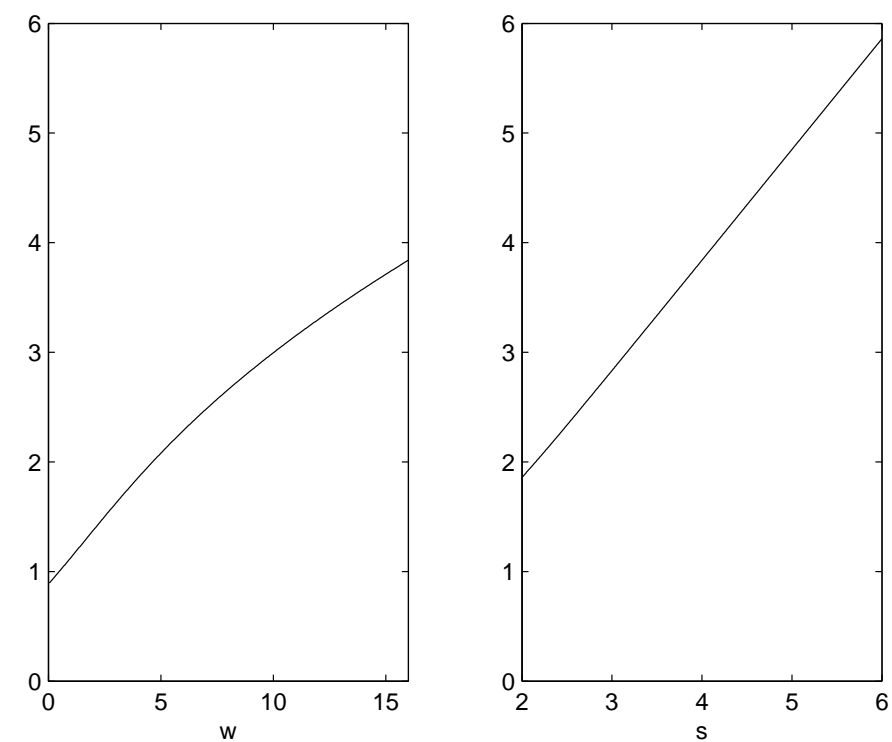

Figure 1: $\operatorname{erfinv}(x) / x$ plotted versus $w$ and $s \equiv \sqrt{w}$. 
Table 2: Three alternatives for single precision approximation of erfinv

\begin{tabular}{|c|c|c|c|}
\hline$w_{1}$ & $p_{1}$ degree & $p_{2}$ degree & tail prob. \\
\hline 5.00 & 8 & 8 & $0.3 \%$ \\
6.25 & 9 & 7 & $0.1 \%$ \\
16.00 & 14 & $\mathrm{n} / \mathrm{a}$ & $0 \%$ \\
\hline
\end{tabular}

The weighting is a standard one under which Chebyshev polynomials are orthogonal, and is introduced to control the errors near the two ends of the interval. A similar construction is used for $p_{2}$.

Table 1 shows the degree of polynomial required for $p_{1}$ and $p_{2}$, to reduce the relative approximation error to less than $10^{-7}$, depending on the choice of the dividing point $w_{1}$. The fourth column gives the approximate probability that a random input $x$, uniformly distributed on $(-1,1)$, will lie in the tail region. Multiplying this by 32 gives the approximate probability that one or more inputs in a CUDA warp of 32 threads will lie in the tail region, and hence that a CUDA implementation will have a divergent warp.

Using $w_{1}=5$, there is roughly a $10 \%$ probability of a divergent warp, but the cost of the central region approximation is the least since it requires only a degree 8 polynomial for $p_{1}$. On the other hand, using $w_{1}=16$, there is no tail region; the central region covers the whole interval. However, $p_{1}$ is now of degree 14 so the cost has increased. The second option uses $w_{1}=6.25$, for which $p_{1}$ needs to be of degree 9 . In this case, there is only a $3 \%$ probability of a divergent warp.

\subsection{Double precision}

The double precision approximation erfinv $v_{D P}$ is defined similarly, but it is defined with up to two tail regions as it needs to extend to $w \approx 36$ to cover the full double precision range for $x$.

$$
\operatorname{erfinv}_{\mathrm{DP}}(x)=\left\{\begin{array}{ccc}
x p_{1}(w), & w \leq w_{1} & \text { central region } \\
x p_{2}(s), & w_{1}<w \leq w_{2} & \text { tail region } 1 \\
x p_{3}(s), & w_{2}<w & \text { tail region } 2
\end{array}\right.
$$

Table 2 shows the degree of polynomial required for $p_{1}, p_{2}$ and $p_{3}$ to 
Table 3: Two alternatives for double precision approximation of erfinv

\begin{tabular}{|c|c|c|c|c|c|}
\hline$w_{1}$ & $w_{2}$ & $p_{1}$ degree & $p_{2}$ degree & $p_{3}$ degree & tail prob. \\
\hline 6.25 & 16.0 & 22 & 18 & 16 & $0.1 \%$ \\
6.25 & 36.0 & 22 & 26 & n/a & $0.1 \%$ \\
\hline
\end{tabular}

reduce the relative approximation error to less than $2 \times 10^{-16}$, depending on the choice of the dividing points $w_{1}$ and $w_{2}$. The last column again gives the approximate probability that a uniformly distributed random input $x$ is not in the central region.

In constructing the weighted least-squares approximation using MAT$\mathrm{LAB}$, variable precision arithmetic (an extended precision feature of the Symbolic Toolbox) is required to evaluate the analytic error function to better than double precision in order to compute the accuracy of the approximation.

\subsection{Floating point error analysis}

In this section we look at the errors due to finite precision arithmetic. The floating point evaluation of $(1-x)(1+x)$ will yield a value equal to

$$
(1-x)(1+x)\left(1+\varepsilon_{1}\right)
$$

where $\varepsilon_{1}$ corresponds to at most $1 \mathrm{ulp}$ (unit of least precision) which is roughly $10^{-7}$ for single precision and $10^{-16}$ for double precision. Computing $w$ by evaluating the log of this will yield, approximately,

$$
w+\varepsilon_{1}+\varepsilon_{2}
$$

where $\varepsilon_{2}$ is the error in evaluating the log function, which is approximately $5 \times 10^{-8} \max (1,3 w)$ when using the CUDA fast single precision function ${ }_{-} \log f()$, and $10^{-16} w$ when using the double precision function $\log ()$.

Computing $p_{1}(w)$ will then yield, approximately

$$
p_{1}(w)\left(1+\left(\varepsilon_{1}+\varepsilon_{2}\right) \frac{p_{1}^{\prime}(w)}{p_{1}(w)}+\varepsilon_{3}\right)
$$

where $\varepsilon_{3}$ is the relative error in evaluating $p_{1}$. The relative error in the 
final product $x p_{1}(w)$ will then be approximately

$$
\left(\varepsilon_{1}+\varepsilon_{2}\right) \frac{p_{1}^{\prime}(w)}{p_{1}(w)}+\varepsilon_{3}+\varepsilon_{4}
$$

where $\varepsilon_{4}$ is again of order $10^{-7}$.

Since $p_{1}(w) \approx 1+0.2 w$, the combined contributions due to $\varepsilon_{1}$ and $\varepsilon_{2}$ are about $1.5 \mathrm{ulp}$ in single precision, and about $0.5 \mathrm{ulp}$ in double precision. The errors due to $\varepsilon_{3}$ and $\varepsilon_{4}$ will each contribute another $0.5 \mathrm{ulp}$. These are in addition to a relative error of roughly $1 \mathrm{ulp}$ due to the approximation of erfinv. Hence, the overall error is likely to be up to 4 ulp for single precision and up to 3 ulp for double precision.

In the tail region, there is an extra step to compute $s=\sqrt{w}$. Since

$$
\sqrt{w+\varepsilon} \approx \sqrt{w}+\frac{\varepsilon}{2 \sqrt{w}}
$$

the value which is computed for $s$ is approximately

$$
s+\frac{1}{2 s}\left(\varepsilon_{1}+\varepsilon_{2}\right)+\varepsilon_{5} s
$$

where $\varepsilon_{5}$ is the relative error in evaluating the square root itself, which for the CUDA implementation is $3 \mathrm{ulp}$ in single precision and less than $0.5 \mathrm{ulp}$ in double precision. Noting also that $p_{2}(s) \approx s$, the relative error in the final result is

$$
\frac{1}{2 w}\left(\varepsilon_{1}+\varepsilon_{2}\right)+\varepsilon_{5}+\varepsilon_{3}+\varepsilon_{4},
$$

In single precision, $\varepsilon_{2} / w$ is about 3 ulp and $\varepsilon_{5}$ is also $3 \mathrm{ulp}$, so the overall error, including the $p_{2}$ approximation error will be about 6 ulp. In double precision, $\varepsilon_{2} / w$ and $\varepsilon_{5}$ are about $1 \mathrm{ulp}$ and $0.5 \mathrm{ulp}$, respectively, so the overall error is 2 to $3 \mathrm{ulp}$.

\section{Performance and accuracy}

Table 4 gives the performance of the first of the new approximations in Tables 2 and 3, compared to the existing ones in CUDA 3.0. Table 5 gives the code for the new single precision approximation. The times are in milliseconds to compute $100 \mathrm{M}$ values, using 28 blocks with 512 threads, and each thread computing 7000 values.

There are two conditions for the tests, "uniform" and "constant". In the uniform case, the inputs $x$ for each warp are spread uniformly in a 
Table 4: Times in milliseconds to compute 100M single precision (SP) and double precision (DP) values using CUDA 3.0 on C1060 and C2050

\begin{tabular}{|c|r|r|r|r|}
\hline & \multicolumn{2}{|c|}{ C1060 } & \multicolumn{2}{c|}{ C2050 } \\
time (ms) & SP & DP & SP & DP \\
\hline uniform, old & 24 & 479 & 31 & 114 \\
uniform, new & 8 & 219 & 10 & 49 \\
constant, old & 8 & 123 & 11 & 30 \\
constant, new & 8 & 213 & 9 & 48 \\
\hline
\end{tabular}

way which ensures $100 \%$ warp divergence for the existing implementation. This worst case scenario is slightly worse than the $87 \%$ divergence which would result from random input data, as discussed in the Introduction. The constant case represents the best case scenario in which all of the threads in each warp use the same input value, though this value is varied during the calculation so that overall each conditional branch is exercised for the appropriate fraction of cases.

The results show a factor 3 or more difference in the performance of the existing implementation on the two test cases. This reflects the penalty of warp divergence, with a cost equal to the sum of all branches which are taken by at least one thread, plus the fact that the execution of the main branch (the one which is taken most often) is significantly less costly because it does not require a $\log$ calculation.

The cost of the new approximations varies very little in the two cases, because even in the "uniform" case almost all warps are non-divergent. On the other hand, all warps have to perform a log calculation, and therefore in double precision the new implementation is slower than the existing one for the constant case.

Regarding accuracy, the maximum error of the new single precision approximation, compared to the existing double precision version, is around $7 \times 10^{-7}$, which is better than the existing single precision version, and the maximum difference between the new and existing double precision implementations is approximately $2 \times 10^{-15}$. 
Table 5: CUDA code for single precision implementation

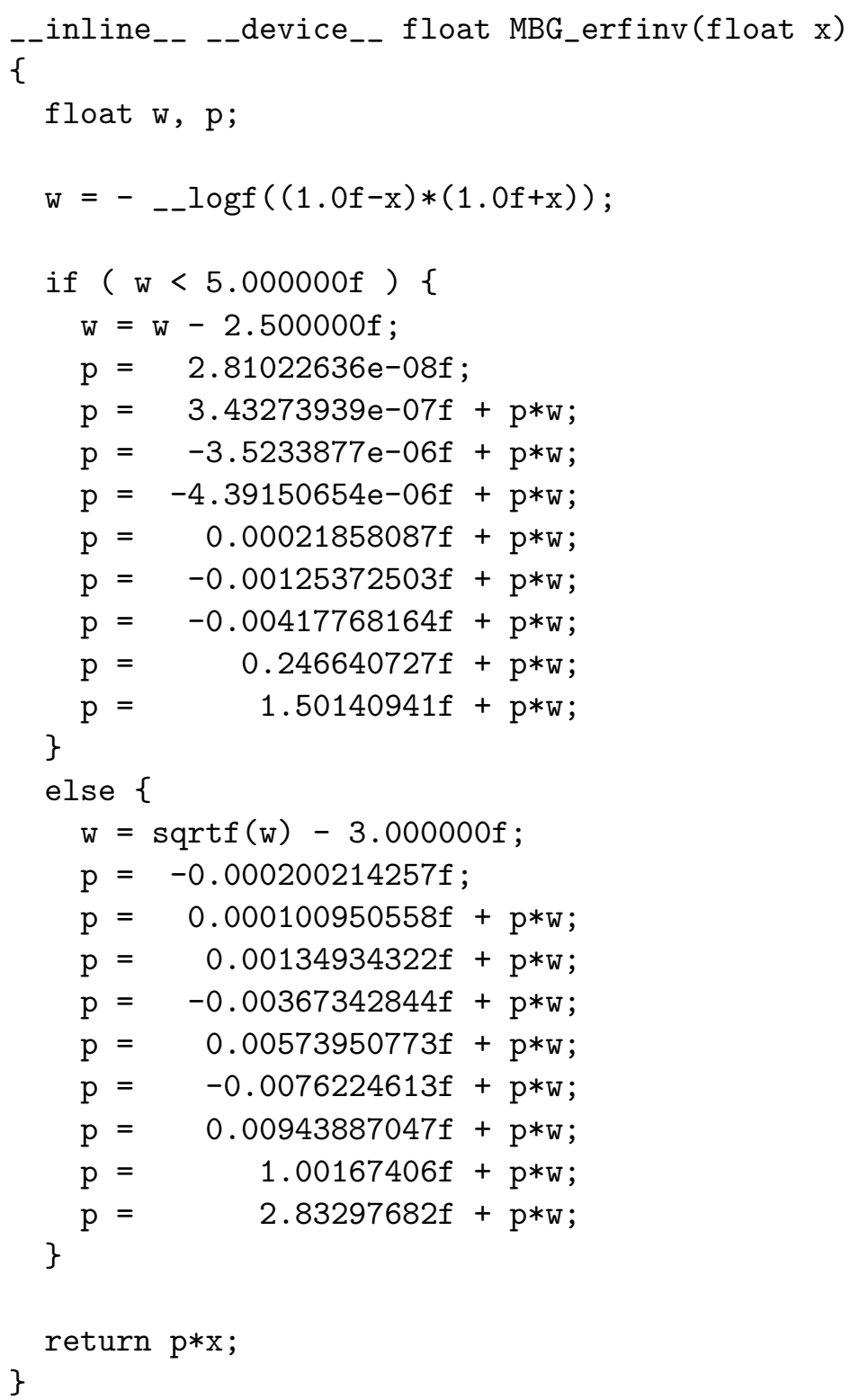




\section{Conclusions}

This chapter illustrates the cost of warp divergence, and the way in which it can sometimes be avoided by re-designing algorithms and approximations which were originally developed for conventional CPUs.

It also illustrates the dilemma which can face library developers. Whether the new double precision approximations are viewed as better than the existing ones depends on how they are likely to be used. For random inputs they are up to 3 times faster, but they can also be slower when the inputs within each warp are all identical, or vary very little.

\section{References}

[BEJ76] J.M. Blair, C.A. Edwards, and J.H. Johnson. Rational Chebyshev approximations for the inverse of the error function. Mathematics of Computation, 30(136):827-830, 1976.

[BTGTW10] T. Bradley, J. du Toit, M. Giles, R. Tong, and P. Woodhams. Parallelisation techniques for random number generators. GPU Computing Gems, Volume 1, Morgan Kaufmann, 2010 .

[Gil10] M.B. Giles. Approximating the erfinv function (source code). http://gpucomputing.net/?q=node/1828, 2010

[Gla04] P. Glasserman. Monte Carlo Methods in Financial Engineering. Springer, New York, 2004.

[NAG09] Numerical Algorithms Group. Numerical Routines for GPUs. http://www.nag.co.uk/numeric/GPUs/, 2009

[Sha09] W.T. Shaw and N. Brickman. Differential equations for Monte Carlo recycling and a GPU-optimized Normal quantile. Working paper, available from arXiv:0901.0638v3, 2009.

[Str68] A.J. Strecok. On the calculation of the inverse of the error function. Mathematics of Computation, 22(101):144-158, 1968.

[Wic88] M.J. Wichura. Algorithm AS 241: the percentage points of the Normal distribution. Applied Statistics, 37(3):477-484, 1988. 